\title{
How does peer teaching compare to faculty teaching? A systematic
}

review and meta-analysis

\section{Abstract}

\section{Purpose}

In undergraduate medical education, peer-teaching has become an established and common method to enhance student learning. Evidence suggests peer-teaching provides learning benefits for both learners and tutors.

We aimed to describe the outcomes for medical students taught by peers through systematic review and meta-analysis of existing literature.

\section{Methods}

Seven databases were searched through 21 terms and their Boolean combinations. Studies reporting knowledge or skills outcomes of students taught by peers compared to those taught by faculty or qualified clinicians were included.

Extracted data on students' knowledge and skills outcomes were synthesised through a random effects model meta-analysis.

Results 
The search yielded 2,292 studies. 553 duplicates and 1,611 irrelevant articles were removed during title-screening. The abstracts of 128 papers were screened against the inclusion and exclusion criteria. Ten studies have been included in the review.

Meta-analyses showed no significant difference in peer-teaching compared to faculty teaching for knowledge or skills outcomes, standardised mean differences were 0.07 (95\% Cl: $-0.07,0.21)$ and 0.11 (95\% Cl: $-0.07,1.29)$ respectively.

\section{Conclusion}

Students taught by peers do not have significantly different outcomes to those taught by faculty. As the process of teaching helps to develop both tutor knowledge and teaching skills, peer-teaching should be supported. 


\section{Introduction}

The methods used to facilitate students' learning in undergraduate medical education are of great importance. One such method, peer teaching, also referred to as peer-assisted learning or near-peer teaching, has become well embedded in education in recent decades (Ten Cate and Durning, 2007b). Its use has spread from general teaching, to higher education, where it has become well established within the medical education community (Topping, 1996). Defined as 'an educational arrangement in which one student teaches one or more fellow students' (Ten Cate and Durning, 2007a), peer teaching offers opportunities for new knowledge and skills to be learned by both tutor (Nestell and Kidd, 2005) and student (Graham, et al., 2008). With increasing student numbers stretching resources further than ever, utilising student teachers, who themselves have recently covered the topic at hand, is now an option for the modern medical school to consider (Ten Cate and Durning, 2007b).

Key to peer teaching are the elements of cognitive and social congruence (Lockspeiser, et al., 2008). Cognitive congruence is the similarity of tutor and student in intellect and thought-processing. Within peer teaching, both learners and teachers are of a similar level educationally leading to greater cognitive congruence compared to faculty-led teaching. This allows peer teachers to express often complex and intimidating topics in ways which learners are better able to understand (Lockspeiser, et al., 2008). This is also assisted by the fact that peer teachers will themselves have recently covered the same material and may be able to express the concepts in a manner to which novices are more receptive. Equally, peer learners and peer teachers 
are expected to be of the same or a similar social standing. As a result of this social congruence, peer teachers seem to express more of an understanding of their learner's needs and concerns, by way of an increased understanding of their social and academic lives (Schmidt and Moust, 1995).

The literature suggests medical students tend to be receptive to peer teaching. Studies have found that peer learners report: a better learning environment and atmosphere (Kassab, et al., 2005), relief of exam-related stress (Kommalage and Thabrew, 2011) and find their colleagues to be a helpful adjunct to the curriculum teaching (Naeger, et al., 2013). The widespread acceptability of peer teaching makes it a virtuous asset within a curriculum.

Another role of peer teaching is to offer 'tomorrow's doctors' the opportunity to practice their teaching skills at an early stage in their careers. As teaching is an integral part of a clinician's job, it is vital that they are effective at imparting knowledge upon both patients and colleagues. Indeed, in the United Kingdom the General Medical Council's guide to 'Good Medical Practice' stresses the importance of competence in the art of teaching (General Medical Council. 2013).

Studies investigating the efficacy of peer teaching have reported measures of students' perceptions of their abilities (Hall, et al., 2013), development of skills (Weyrich, et al., 2009) and knowledge (Hendelman and Boss, 1986).

Findings suggest peer teaching leads to an improvement in students' selfreported confidence, and has been shown to increase knowledge and skills. Subjective measures of peer teaching support social and cognitive 
congruence hypotheses and provide assurance that peer teaching is well received. Furthermore, objective measurements of efficacy provide a valid evidence base for peer teachings' continued use.

However, to date, there has been no full analysis of the evidence available on the impact of peer teaching with respect to medical students' skills and knowledge. With peer teaching becoming more and more common, it is crucial to understanding the value of these learning activities.

It is reasonable to assume that any teaching intervention will be more effective at developing learners' skills or knowledge than no intervention. Therefore, the positive results of non-comparative pre- post- studies of peer teaching are unsurprising. Of greater interest are studies that compare different modes of teaching and learning, in order to determine if either is more effective.

This review, therefore, intends to describe the knowledge and skills outcomes for undergraduate medical students taught through peer teaching compared to those taught by faculty. 


\section{Methods}

Review question

How are the knowledge and skills outcomes of undergraduate medical students taught by peers different to those taught by faculty?

Search strategy

Electronic searches of 16 terms and their Boolean combinations were conducted through seven databases (Education Resources Information Centre (ERIC), Medline, Web of Science, EMBASE, Cumulative Index to Nursing and Allied Health (CINAHL), British Education Index, Australian Education Index,).

The search was performed on $16^{\text {th }}$ October 2013 using the following strategy:

1. "peer teach*".ti,ab;

2. "near peer teach*".ti,ab;

3. "peer assisted learning".ti,ab;

4. "student teach*".ti,ab;

5. "peer to peer".ti,ab;

6. "peer tutor\$".ti,ab;

7. 1 OR 2 OR 3 OR 4 OR 5 OR 6;

8. undergraduat*.ti,ab;

9. initial.ti,ab;

10. universit*.ti,ab;

11. student\$.ti,ab;

12. school\$.ti,ab;

13. bachelor\$.ti,ab;

14. Degree.ti,ab; 
15. graduate.ti,ab;

16. "pre-registration".ti,ab;

17. 8 OR 9 OR 10 OR 11 OR 12 OR 13 OR 14 OR 15 OR 16;

18. medic $^{\star} . \mathrm{ti}, \mathrm{ab}$;

19. "medical education".ti,ab;

20. 18 OR 19;

21. 7 AND 17 AND 20;

The reference lists of all papers included in full paper review were screened for additional relevant papers. Finally, four key journals (Academic Medicine, Medical Education, Medical Teacher, The Clinical Teacher) were hand searched through the last ten years (2004-2013) for further relevant papers. Selection criteria

The criteria for inclusion in this systematic review were as follows:

Population: Undergraduate medical students.

Intervention: Peer teaching

Comparison: Faculty teaching

Outcomes: Knowledge or skills outcomes measured through objective assessment.

Study design: Comparative.

Screening and selection of studies

Article titles were screened by one reviewer (BD), duplicates and papers that were clearly not related to peer teaching were excluded. Abstracts were independently screened against inclusion and exclusion criteria by two reviewers (PQ \& $B D)$. In the case of disagreement the opinion of a third 
reviewer (ELR) was sought. The full papers for all remaining citations were retrieved and reviewed against inclusion and exclusion criteria by one reviewer (PQ, BD or VF). Studies meeting inclusion criteria progressed to data extraction and assessment of methodological quality.

\section{Pilot study}

In order to refine the review question and ensure reviewer consistency in data extraction and assessment of methodological quality, a pilot study was conducted. All reviewers independently reviewed five articles and met to discuss discrepancies and to finalise the data extraction form.

\section{Data extraction}

Two reviewers (ELR and one of $P Q, B D$ or VF) independently extracted data from each article, including details regarding study methodology, population studied, peer-teaching intervention, comparison, outcomes measured and findings.

\section{Assessment of methodological quality}

The methodological quality of included studies was rated using the Medical Education Research Study Quality Instrument (MERSQI) (Read, et al., 2007). The MERSQI is a validated tool consisting of ten items in six domains; study design, sampling, type of data, validity of evaluation instrument, data analysis, and outcomes.

Assessment of risk of bias

Risk of bias of included studies was determined using the Cochrane collaboration risk of bias assessment tool (Higgins and Green, 2011). A judgement of 'low risk of bias', 'unclear risk of bias', or 'high risk of bias' has been given for each source of bias for each study accompanied by supporting 
statements. The sources of bias considered were: random sequence generation (selection bias), allocation concealment, (selection bias), blinding of participant and personnel (performance bias), blinding of outcome assessment (detection bias) and incomplete outcome data (attrition bias). Data synthesis

Data regarding students' outcomes were synthesised using random-effects model meta-analysis in Comprehensive Meta Analysis (Biostat inc, New Jersey, USA). 


\section{Results}

\section{Search results}

The database search yielded 2,292 articles. Reference screening and hand searching yielded an additional 72 and six articles respectively. 553 duplicates were removed, and 1,611 articles were excluded during title screening. 206 abstracts were reviewed against inclusion and exclusion criteria, 110 were excluded. 96 full papers were screened against inclusion criteria, 86 were excluded. The remaining ten papers were included in the review. Figure 1 illustrates the flowchart of included papers.

\section{Methodological quality}

The mean (SD, range) MERSQI score for included studies was $13.25(0.65$, 12.5 to 15.5 ) out of 18 (table 2 ). The range of scores is low as in order to meet inclusion criteria studies needed to have objective measures of knowledge or skills. All studies utilised appropriate, beyond descriptive, analyses. No study sampled from more than one institution. The majority of the variability in MERSQI scores arose from the 'validity of evaluation instrument' domain, where the mean (SD) score was $1.10(0.92)$ out of a possible 3 .

\section{Risk of bias}

The risk of bias and justification for individual studies is detailed in table 3 , the overall risk of bias for included studies is illustrated in figure 2. Due to the nature of the intervention and comparison, study participants could not be blinded in any of the studies. No studies reported participant attrition as the assessments following the intervention appeared to be compulsory. Studies inadequately reported the process of randomisation of participants. However, 
as all participants would be recruited and randomised at the same time, the risk of bias from random sequence generation was considered to be low. The greatest area of potential bias in these studies was in blinding of outcome assessors; five (50\%) of included studies reported assessors being blinded to participants allocation, however the other five (50\%) did not provide any details.

\section{Summary of included papers}

Methods, population, intervention and comparator, outcomes, and findings for each included study are detailed in table 1. The ten studies recruited a total (mean, range) of $1,300(130,39$ to 292$)$ participants. All included studies assessed skills outcomes, five (50\%) also assessed knowledge. The studies were conducted in five countries (USA, UK, Australia, Germany, and Nigeria) with Germany accounting for half $(n=5)$ of the studies.

Knowledge:

Only one study reported a significant difference between peer-taught and faculty-taught students' knowledge; favouring peer teaching. The pooled effect favours peer-teaching, but does not reach significance (standardised mean difference $(\mathrm{SMD})=0.07, \mathrm{p}=0.32)$ (figure 3 ).

Skills:

For skills outcomes, again only one study reported significantly different outcomes between peer-taught and faculty-taught students (again in favour of peer-teaching). The pooled effect favoured peer teaching but again failed to reach significance $(\mathrm{SMD}=0.11, \mathrm{p}=0.24)$ (figure 4$)$. 


\section{Discussion}

This study aimed to synthesise existing evidence of medical students' knowledge and skills outcomes when taught by faculty members compared to those taught by student peers. Of the 2,292 studies screened, we identified and included ten that compared the two groups and used objective outcome (assessment) measures. There appears to be no significant difference in knowledge or skills outcomes of students taught by either student peers or faculty members.

That there is no difference in students' outcomes in the included papers does not suggest that all students would be as effective as staff at teaching any topic in the undergraduate medical curriculum. Eight (80\%) of the included studies investigated teaching of physical examination or communication skills, while only two studies researched basic or clinical sciences. It may be that students are equally effective at teaching communication, clinicial and procedural skills, but that basic or clinical sciences teaching might require even greater expertise. Nevertheless, this meta-analysis demonstrates that student peers are as effective at teaching these certain topics as faculty. Considering the reported acceptability to the peers they teach (Kassab, et al., 2005), the educational benefit to the peer tutors (Nestell and Kidd, 2005), and the necessity for undergraduate students to develop competency in teaching (General Medical Council, 2009; General Medical Council, 2013), we suggest it is appropriate for students to deliver teaching to their peers.

Careful thought should be given to how peer teaching is implemented and monitored within medical schools and will depend on the aim of peer teaching within a given school, whether to alleviate pressure on faculty and resources, 
or purely for the educational development of the peer tutors. Nestel and Kidd report using peer teachers to replace faculty in communication skills training due to a shortage in resources (Nestell and Kidd, 2003). These peer teachers attended a teacher-training workshop and received remuneration for the sessions they delivered.

In other schools students deliver extracurricular teaching as an adjunct to faculty teaching (Naeger, et al., 2013). Peer teaching in addition to faculty teaching is likely to be better than faculty teaching alone, and will still offer the educational benefit to the peer teachers.

Regardless of whether peer-teaching forms part of a school's curriculum or is supplementary, it seems sensible that peer tutors should receive some form of instruction on teaching theory and methods. Though more rigorous quality assurance would be necessary if the sessions form part of the school's formal curriculum. A recent review identified peer teaching programmes, teaching workshops and community outreach programmes as the three leading initiatives for developing students' teaching skills (Marton, et al., 2015). Peer observation of peer teaching may also be valuable for ongoing development of teaching skills (Rees, et al., 2015).

Within each of the studies included students volunteered to teach. Whilst it is appropriate that those with an interest in teaching have an opportunity to engage, it raises the question of whether all students should be expected to teach or if it should remain optional. Some institutions select students to be peer tutors on the basis of previous academic performance (Sobral, 2002) or performance in a personal interview (Weyrich, et al., 2008)

Strengths and limitations 
To our knowledge, this is the first meta analysis of students outcomes after peer teaching versus faculty teaching. We developed a comprehensive, sensitive search strategy to identify as many potentially relevant articles as possible, and then screened each against inclusion criteria. We undertook a pilot study to ensure that reviewers were extracting data and assessing methodological quality consistently.

As a review this study is limited by the availability of published literature.

Despite employing a comprehensive search strategy, few articles that met our inclusion criteria were identified. The meta analysis is therefore based on the outcomes of only 1300 students in ten studies. Furthermore, there was considerable variability in the studies included in terms of the content taught, the educational level of the tutors and the outcome assessment measures. The findings of this meta analysis should therefore be interpreted with this educational heterogeneity in mind. This heterogeneity notwithstanding, the analysis suggests there is no clear and significant difference in students' outcomes when taught by either group.

Areas for future research

The findings of this analysis have demonstrated that medical students, with limited experience and training in both medicine and the art of teaching, may function as effectively as more experienced faculty teachers. Future research into how peer teachers are able to bridge this gap is an interesting proposition.

An area of interest for future study would be the topics within undergraduate medical curricula that are most appropriate for peer teaching. The studies included in this review have looked at both skills and knowledge taught 
through peer to peer learning. However, no studies to date have compared peer teaching in different aspects of the medical curricula effectively. The findings of this research would allow the benefits of peer teaching to be maximised, through use in the areas in which it is most effective. Moreover, detriments would be minimised as faculty teaching can be focussed in areas where peer-led tuition is less useful.

In the literature regarding benefits to teachers from peer learning, many studies are limited by selection bias due to the fact that peer teachers are often volunteers and are, therefore, more likely to be eager students with better outcomes. However, as advantages of peer teaching to the tutor have been demonstrated (Nestell and Kidd, 2005), it is possible that it may be an effective educational strategy to encourage less well-performing students to engage with this process. Contrary to this, it may be argued that this is a less valuable way of approaching peer teaching from the perspective of the learner, due to the fact that less able students are not as likely as their more adept colleagues to be able to understand and convey the information required. Further investigation into this with a comparison of outcomes from groups led by students of varying ability, would be enlightening and allow better utilization of peer teaching.

A final topic for future debate is that of quality assurance. Within many of the studies included in this article, faculty were able to review learning plans and resources in order to ensure their accuracy. It may be valuable to take this a step further, with the introduction of senior supervision in peer-taught sessions. This may provide an anxious tutor with a safety net for difficult questions, and allows the associated institution to ensure appropriate 
teaching practice. However, this risks disrupting the educational environment generated by the informal nature of peer teaching, and may therefore be disadvantageous.

\section{Practice points}

- Experience of teaching peers at an undergraduate level enables students to develop their teaching skills early.

- There appears to be no difference in knowledge or skills outcomes for students taught by peers compared to those taught by faculty members.

- Peer teaching should be supported at least in the form of extracurricular teaching.

- Mechanisms may have to be adopted to ensure the quality of peer teaching. 
Acknowledgements:

None

Funding:

None

Other disclosures:

None

Ethical approval:

Not required

Disclaimer:

None

Previous presentations:

Findings from this study were presented as an oral presentation at the 2014 Annual Scientific Meeting of the Association for The Study of Medical

Education (ASME) in Brighton, UK on 17 $7^{\text {th }}$ July 2014. 


\section{References}

Buckley S and Zamora J. 2007. Effects of participation in a cross year peer tutoring programme in clinical examination skills on volunteer tutors' skills and attitudes towards teachers and teaching. BMC Medical Education. 7; 20.

Büscher R, Weber D, Büscher A, Hölscher M, Pohlhuis S, Groes B and Hoyer PF. 2013. Evaluation of the peer teaching program at the University Children's Hospital Essen - a single center experience. GMS Z Med Ausbild. 30; doi: 10.3205/zma000868.

Coleman MM, Blatt B and Greenberg L. 2012. Preparing students to be academicians: A national student-led summer program in teaching, leadership, scholarship and academic medical career-building. Academic Medicine. 87; 1734-1741.

General Medical Council. 2009. Tomorrow's doctors. London: GMC

General Medical Council. 2013. Good medical practice. London: GMC

Graham K, Burke JM and Field M. 2008. Undergraduate rheumatology: Can peer-assisted learning by medical students deliver equivalent training to that provided by specialist staff? Rheumatology. 47; 652-655.

Haist SA, Wilson JF, Brigham NL, Fosson S,E. and Blue AV. 1998.

Comparing fourth-year medical students with faculty in the teaching of physical examination skills to first -year students. Academic Medicine. 73; 198--200. 
Haist SA, Wilson JF, Fosson SE and Brigham NL. 1997. Are fourth-year medical students efective teachers of the physical examination to first-year medical students? Journal of General Internal Medicine. 12; 177-181.

Hall L, Lewis M and Border S. 2013. Near-peer teaching in clinical neuroanatomy. The Clinical Teacher. 10; 230-235.

Heckmann JG, Dutsch M, Rauch C, Lang C, Weih M and Schwab S. 2008. Effects of peer-assisted training during the neurology clerkship: A randomized controlled trial. European Journal of Neurology. 15; 1365-1370.

Hendelman W and Boss M. 1986. Reciprocal peer teaching by medical students in the gross anatomy laboratory. Journal of Medical education. 61; 674-680.

Higgins J, Green S, eds. 2011. Cochrane handbook for systematic reviews of interventions version 5.1.0. The Cochrane Collaboration.

Hudson JN and Tonkin AL. 2008. Clinical skills education: Outcomes of relationships between junior medical students, senior peers and simulated patients. Medical Education. 42; 901-908.

Kassab S, Abu-Hijleh MF, Al-Shboul Q and Hamdy H. 2005. Student-led tutorials in problem-based learning: Educational outcomes and students' perceptions. Medical Teacher. 27; 521-526.

Knobe M, Holschen M, Mooij SC, Sellei RM, Münker R, Antony P, Pfeifer R, Drescher W and Pape HC. 2012. Knowledge transfer of spinal manipulation 
skills by student-teachers: a randomised controlled trial. Eur Spine J. 21; $992-$ 998.

Knobe M, Münker R, Sellei RM, Holschen M, Mooij SC, Schmidt-Rohlfing B, Niethard FU and Pape HC. 2010. Peer teaching: a randomised controlled trial using student-teachers to teach musculoskeletal ultrasound. Med Educ. $44 ; 148-155$.

Kommalage M and Thabrew H. 2011. Student-led peer-assisted learning: The kuppi experience at the medical school of the university of ruhuna in sri lanka. education for health. Education for Health. 24; 516

Lockspeiser T, O'Sullivan P, Teherani A and Muller J. 2008. Understanding the experience of being taught by peers: The value of social and cognitive congruence. Advances in Health Sciences Education. 13; 361-372.

Marton GE, McCullough B and Ramnanan CJ. 2015. A review of teaching skills development programmes for medical students. Medical Education. 49; $149-160$

Naeger DM, Conrad M, Nguyen J, Kohi MP and Webb EM. 2013. Students teaching students: Evaluation of a "Near-peer" teaching experience. Academic Radiology. 20; 1177-1182.

Nestel D and Kidd J. 2003. Peer tutoring in patient-centred interviewing skills: Experience of a project for first-year students. Medical Teacher. 25; 398-403. 
Nestell D and Kidd J. 2005. Peer assisted learning in patient-centred interviewing: The impact on student tutors. Medical Teacher. 27; 439-444.

Nnodim JO. 1997. A controlled trial of peer-teaching in practical gross anatomy. Clinical Anatomy. 10; 112-117.

Pasquale SJ and Cukor J. 2007. Collaboration of junior students and residents in a teacher course for senior medical students. Medical teacher. 29; 572-576.

Reed DA, Cook DA, Beckman TJ, Levine RB, Kern DE and Wright SM. 2007. Association between funding and quality of published medical education research. JAMA. 298; 1002-1009.

Rees EL, Davies B and Eastwood M. 2015. Developing students' teaching through peer observation and feedback. Perspectives on Medical Education. $4 ; 268-271$

Schmidt HG and Moust JH. 1995. What makes a tutor effective? A structural equations modelling approach to learning in problem-based curricula. Academic Medicine. 70; 708-714.

Sobral DT. 2002. Cross-year peer tutoring experience in a medical school: conditions and outcomes for student tutors. Medical Education. 36; 10641070

Ten Cate O and Durning S. 2007a. Dimensions and psychology of peer teaching in medical education. Med Teach. 29; 546-552 
Ten Cate O and Durning S. 2007b. Peer teaching in medical education: Twelve reasons to move from theory to practice. Med Teach. 29; 591-599.

Topping KJ. 1996. The effectiveness of peer tutoring in further and higher education: A typology and review of the literature. High Educ. 32; 321-345

Weyrich P, Celebi N, Schrauth M, Moltner A, Lammerding-Koppel M and Nikendei C. 2009. Peer-assisted versus faculty staff-led skills laboratory training: A randomised controlled trial. Medical Education. 43; 113-120.

Weyrich P, Schrauth M, Kraus B, Habermehl D, Netzhammer N, Zipfel S, Junger J, Riessen R and Nikendei C. 2008. Undegraduate technical skills training guided by student tutors-analysis of tuotrs' attitudes, tutees' acceptance and learning progress in an innovative teaching model 


\section{Figures and tables:}

Figure 1 - Study inclusion flowchart

Table 1 - Details of included studies

Table 2 - Methodological quality of included studies

Table 3 - Risk of bias

Figure 2 - Summary of risk of bias

Figure 3 - Meta analysis of knowledge outcomes

Figure 4 - Meta analysis of skills outcomes 